\title{
BMJ A longitudinal, qualitative and Open quantitative exploration of daily life and need for rehabilitation among patients with high-grade gliomas and their caregivers
}

\author{
K Piil, ${ }^{1,2} \mathrm{M}$ Jarden, ${ }^{2} \mathrm{~J}$ Jakobsen, ${ }^{3} \mathrm{~K}$ Bang Christensen, ${ }^{4} \mathrm{M}$ Juhler ${ }^{1,5}$
}

To cite: Piil K, Jarden M, Jakobsen J, et al. A longitudinal, qualitative and quantitative exploration of daily life and need for rehabilitation among patients with high-grade gliomas and their caregivers. BMJ Open 2013;3:e003183. doi:10.1136/bmjopen-2013003183

- Prepublication history for this paper is available online. To view these files please visit the journal online (http://dx.doi.org/10.1136/ bmjopen-2013-003183).

Received 8 May 2013 Revised 11 June 2013 Accepted 14 June 2013

This final article is available for use under the terms of the Creative Commons Attribution Non-Commercial 3.0 Licence; see http://bmjopen.bmj.com

For numbered affiliations see end of article.

\section{Correspondence to} K Piil;

Karin.piil@rh.regionh.dk

\section{ABSTRACT}

Introduction: High-grade gliomas (HGGs) are the most malignant type of brain tumours. The 5 -year survival is $10 \%$ and a significant part of the ongoing research aims to increase survival through surgical and oncological treatments. Accordingly, there is an increasing need for investigating the $H G G$ trajectory in order to recommend specific guidelines for rehabilitative and supportive interventions.

Method and analysis: This study protocol (phase I) describes a longitudinal, qualitative, explorative and descriptive interview study of the life situation and need for rehabilitation among patients and their caregivers and a quantitative evaluation of health-related quality of life. Qualitative and quantitative data are collected in parallel, analysed separately and then merged. The finding of this study will, together with the existing literature, form the background for phase II, which is a feasibility study with a pre-experimental one-group design testing a rehabilitative and supportive intervention programme. The aim of this paper was to describe the design of an upcoming study. Interviews with 30 patients and 30 caregivers will provide information about how the life situation is experienced during the first year after being diagnosed with HGG. Quantitative measurements of quality of life, well-being and physical activity will provide additional information. More precisely, both qualitative and quantitative data will support the planning of the programme regarding the type of intervention(s), with or without supervision, the appropriate time along the trajectory, frequency, localisation, endpoint measurements and eligible patients and/or caregivers.

Ethics and dissemination: According to the Research Ethics Committee, approval is not needed for phase I as it is a non-intervention part of the study. Ethical approval of phase II will be sought at the time where the content of the intervention programme has been developed. Dissemination will occur through presentation and findings will be published in peer-reviewed journals.

\section{ARTICLE SUMMARY}

Article focus

- This protocol article describes a mixed-method longitudinal study of the life situation among patients newly diagnosed with high-grade glioma (HGG) and their caregivers. The supportive, rehabilitative and palliative needs and preferences during the first year after diagnosis will be explored in order to develop a rehabilitation programme that targets these special needs.

\section{Key messages}

- The literature that addresses the supportive, rehabilitative and palliative needs still remains limited and reflects a gap in the existing knowledge regarding the breadth of needs across the disease and treatment trajectory for patients with HGG and their caregivers.

- Patients and their caregivers might have different needs for support, rehabilitation and palliation at various stages of the disease and treatment trajectory.

- Few intervention studies have been conducted in patients with $H G G$, leaving the present evidence for specific guidelines for the supportive, rehabilitative and palliative interventions poor.

Strengths and limitations of this study

- A key strength of this study is its mixed-methods longitudinal design including the perspectives of both the patient and their caregiver. A potential limitation is the uncertainty of recruitment immediately after surgery (1-2 days postoperative), as well as the risk of dropout due to the nonadherence, inability to comply or death in patients with $\mathrm{HGG}$ and their caregivers during longitudinal research.

\section{BACKGROUND}

Primary brain cancer accounts for $2 \%$ of all cancer cases. ${ }^{1}$ High-grade glioma (HGG) is the most malignant type of brain tumour 
(WHO classification grade III/IV) occurring most frequently in people aged $45-70$ years. $^{2}$ Median survival time is $12-15$ months $^{3}$ and the 5-year survival is $10 \% .^{4}$ A significant part of the ongoing research aims to increase survival through surgical and oncological treatments, ${ }^{5}$ and this has resulted in a gradual improvement in prognosis over the past three decades. Accordingly, there is an increasing need for investigation of the HGG disease and treatment trajectory in order to recommend specific guidelines for the supportive, rehabilitative and palliative interventions.

The current standard combined modality treatment for HGG consists of surgery followed by daily outpatient radiation therapy and 6 weeks of concomitant chemotherapy (temozolomide). After a pause of 23 days, a new cycle of chemotherapy is repeated. A total of six cycles of adjuvant temozolomide is administered to each patient. A curative treatment does not exist, but radiation and chemotherapy have a life-prolonging effect. ${ }^{5}$ Treatment response is evaluated by brain imaging and typically carried out prior to the third and sixth treatment cycles (see figure 1). In case of tumour progression, suitable patients can be offered reoperation or treatment with an angiogenesis inhibitor (Bevacizumab) in combination with chemotherapy (Irinotecan).

Patients with HGG differ from other patients with cancer in that these tumours cause damage to brain function and the nervous system. ${ }^{6}{ }^{7}$ Being diagnosed with HGG has been shown to result in a significant symptom burden related to both the disease and the treatment. ${ }^{8-15}$ All types of cerebral symptoms can occur including global cerebral symptoms (fatigue, nausea, headaches, confusion), focal symptoms (hemiparesis, seizures and speech difficulties), neurocognitive deficits (aphasia, impaired attention, concentration difficulties, reduced short-term memory, personality changes) and emotional symptoms such as depression and anxiety. ${ }^{16-20}$ Several of these symptoms have been shown to influence both survival and quality of life (QOL). Impairment of the physical condition and fatigue ${ }^{21-24}$ are prominent symptoms which reduce QOL during the postoperative period. ${ }^{25}$ The degree of cognitive impairment and the grade of malignancy exert a negative influence on the patient's perception of QOL. ${ }^{26-29}$ One study found that the patient's level of independence was an indicator of survival. ${ }^{30}$ In line with that, exercise behaviour has been found to be a strong independent predictor of survival. ${ }^{31}$

Glucocorticoids are commonly administered in palliative treatment to decrease tumour or radiationassociated oedema. However, steroids cause significant toxicity with prolonged use, ${ }^{32}$ and frequently result in systemic and cerebral side effects, for example, excitement, insomnia, hyperglycaemia, gastric disturbances, osteoporosis and loss of muscle strength, ${ }^{33-35}$ which may also influence QOL.

Previous studies show that social relationships between the patient and their relatives change as the relative becomes the nearest caregiver. The caregiver is a key resource for the patient, often taking necessary responsibility for the patient throughout the course of treatments. However, caregivers are overwhelmed by the new situation often requiring support. ${ }^{36}$ Several studies show that the QOL of the caregiver is negatively affected by the patient's disease. ${ }^{36-38}$ Both the patient and the caregiver experience agony, ${ }^{39}$ and have spiritual and existential considerations. ${ }^{40}{ }^{41}$ Most caregivers find that their relation to the workforce is adversely affected ${ }^{42}$ and they experience stress. ${ }^{43-46}$ Very little is known about how the patients and their caregivers experience the disease and treatment across the trajectory. It is unclear which needs are present at different stages, how these needs are best addressed and by whom. Few intervention studies have been conducted and as a consequence, no guidelines regarding the supportive, rehabilitative and palliative interventions exist, and the evidence for the development of such specific guidelines is poor.

\section{Rationale for the study protocol}

Patients with HGG are not well represented in studies that include other types of brain cancer.

There is a lack of non-pharmacological intervention studies focusing exclusively on patients with HGG and their caregivers. The literature addressing the supportive,
Figure 1 Treatment trajectory and study evaluation (phase I).

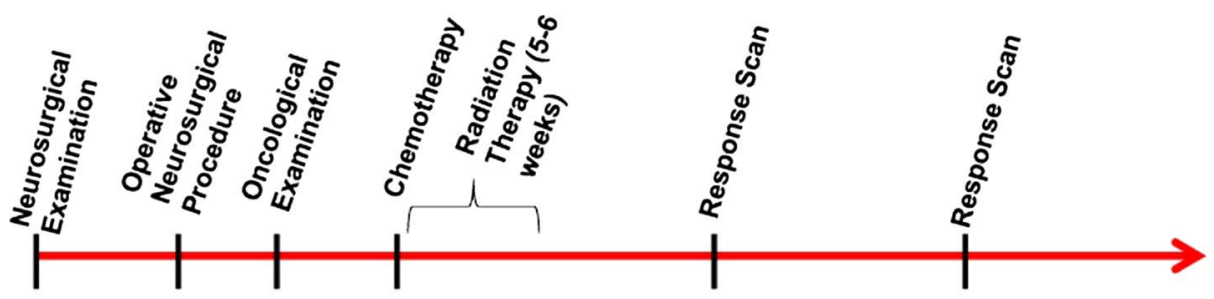

Study Evaluation:

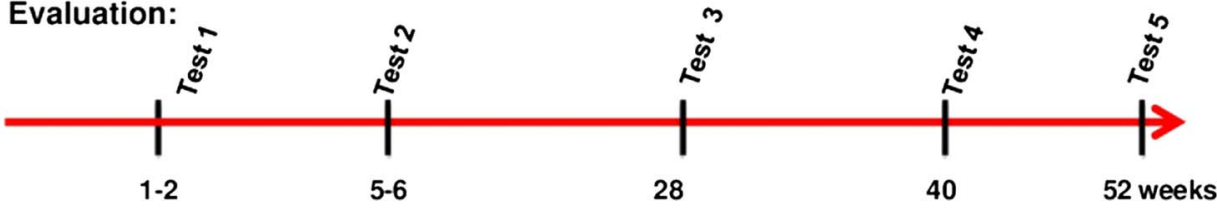


rehabilitative and palliative needs remains limited and diffuse $^{47}$ and reflects a gap in the existing knowledge regarding the breadth of needs across the disease and treatment trajectory for patients with HGG and their caregivers. Further, combining qualitative interviews and quantitative patient-reported outcomes to study QOL, emotional well-being and level of physical activity has not yet been carried out in patients with HGG and their caregivers applying a longitudinal design. The reason for converging the two forms of data is to bring greater insight into the aims of this study than would be obtained by either type of data separately. ${ }^{48}$

\section{Aim}

The study comprises two consecutive phases. The primary aim of phase I was to explore the life situation, needs and experiences across a 1-year disease and treatment continuum in patients newly diagnosed with HGG and their caregivers. Furthermore, the preferences for the supportive, rehabilitative and palliative interventions during and after surgical and medical treatment for HGG will be investigated. Specifically, the aims were

- To gain insight into the life situation of patients and their caregivers during the first year after diagnosis.

- To systematically assess the patient's need for support, rehabilitation and palliation throughout the course of the disease.

- To describe how symptoms are experienced and managed.

- To elucidate the resources of the caregivers and any independent needs related to the patient's disease.

- To gain an understanding of what a comprehensive survivorship programme should comprise.

- To develop a targeted rehabilitation programme for patients with HGG and their caregivers.

The aim of phase II was to study the feasibility of a rehabilitation programme designed from the existing literature and from the experiences of the patients and their caregivers obtained in the present study (phase I). The pilot study (phase II) will investigate the feasibility, adherence, safety and preliminary benefits of recruiting patients with HGG and their caregivers for participation in the supportive, rehabilitative and palliative interventions. The precise study design of phase II is dependent on the results of phase I and will therefore not be described in further detail in this article.

\section{METHODS}

\section{Study participants and sampling}

Patients will be recruited by a criterion sampling strategy. The criteria includes a diagnosis of HGG (WHO classification grade III/IV) in persons $>18$ years of age who are capable of speaking and understanding Danish and who have a Karnofsky performance score (KPS) $>60$ at baseline. The rationale for KPS $>60$ is to assure recruitment of the best informants. Since the present study requires active and independent participation during the interview sessions and in filling out the questionnaires, KPS $>60$ is considered a necessary requirement for adequate participation. Whether patients with cognitive deficits will be able to participate in the study will depend on the judgements of clinical specialists such as the neurosurgeon, the neuropsychologist and the nurses. Caregivers are eligible if they are named by the patient as the most involved caregiver, and will provide written informed consent. To ensure adequate sample size and optimal recruitment, the following has been taken into consideration: first, in order to ascertain data saturation at each test point during the entire course of the study (1 year), the sample size at baseline takes into account an expected drop-out rate of $50 \%$ at the final test (12 months). The number of newly diagnosed patients with HGG (WHO classification grades III and IV) at Rigshospitalet is approximately 90 during a 6 -month period. Based on an estimated reduction of $50 \%$ in the number of patients at the final test ( 1 year from baseline), the necessary sample size is estimated to be 30 patients and 30 caregivers. The expected drop-out rate of $50 \%$ is based on the poor prognosis of a median survival time at $12-15$ months. ${ }^{3}$ Furthermore, it is unknown if participants will experience research participation over 1 year to be too burdensome. It is a risk that a proportion of patients and/or caregivers will dropout or be non-respondents at any of the five test time point. Still, it was decided not to increase the sample size by recruiting new patients/caregivers during the study period due to dropouts, as the study would then lose its longitudinal design for each participant. ${ }^{49}$ Second, recruiting patients with or without caregivers and caregivers with or without patients will be accepted.

\section{Recruitment}

The investigator (neuro-oncology nurse specialist) will consecutively identify eligible patients and ask for participation 1-2 days after the operation. The rationale for this time frame is that patients are often discharged 1-2 days after surgery, and therefore, the process of screening, recruiting and informing potential participants in person is a priority of the investigator for the main purpose of fulfilling eligibility and selection requirements, and assessing performance status. Further, since data generation (interviews and questionnaires) will not be face-to-face, but provided by telephone, the initial meeting with the patient enables personal contact. Patients and caregivers might be in a state of initial shock and distress at this time, ${ }^{50}$ which calls for careful consideration when recruiting this group of patients and their caregivers to research, for example, it is important to limit participants' response burden. Patient motivation has been found to be of considerable importance in the successful collection of QOL data. ${ }^{51}$ Patients and caregivers will be informed and asked to sign the consent form before the patient is discharged from the hospital. 


\section{Design}

This is a convergent parallel design. ${ }^{48}$ Both qualitative and quantitative methods will be applied in this study. The approach for data collection in this mixed methods study is a concurrent approach, ${ }^{48}{ }^{52}$ where qualitative and quantitative data are collected in parallel, analysed separately and then merged. ${ }^{48}$ The quantitative data seek to demonstrate that the variables are systematically related and the qualitative data seek to provide insight into how they are related. ${ }^{52} \mathrm{~A}$ qualitative approach allows the participants to express and describe experiences and thoughts regarding their new life situation in their own words. The quantitative patient-reported psychometric questionnaires will provide additional information to the findings obtained in the interviews. The dominant status relies on the qualitative data as the serial interviews are emphasised as the best suited method to address the primary aim of this study. ${ }^{49}$ The quantitative data are viewed as a useful supplementary data source. The qualitative and quantitative data will be integrated during the interpretation and the findings will draw inferences using both approaches. The feasibility of using questionnaires in this longitudinal design will be explored.

\section{Theoretical framework}

Owing to the complexity of the disease, its treatment and an uncertain life situation, the theoretical framework for this study is the biopsychosocial model. ${ }^{27}$ The biopsychosocial perspective suggests that the biological, psychological and social factors all play a significant role for human functions related to the disease. The interview guide is constructed in accordance with this perspective.

\section{Qualitative data}

The semistructured interviews ${ }^{53}$ will elucidate the subjective experience of having been diagnosed with and treated for HGG and the experience of being a caregiver. In addition to the biopsychosocial perspective, professional knowledge of the disease and course of treatment are incorporated in the construction of the semistructured interview guide. The semistructured interview guide has been developed in accordance with the disease and treatment conditions, for example, surgery (test 1), oncological treatment (test 2), treatment response scans and oncological treatment (tests 3 and 4) and life after standard treatment (test 5). The interview topics for patients appear in table 1 and for caregivers in table 2.

After the initial face-to-face encounter during recruitment, five separate interviews with the patient and their caregiver will take place over the course of a year. The interviews will be conducted by telephone. The decision to choose telephone interviews as the most appropriate method for data collection at multiple time points is based on evidence in the literature. Telephone interviewing is not only an inexpensive and time-saving method, ${ }^{54}{ }^{55}$ but also it has been evaluated as a valid and useful method in qualitative research. ${ }^{56}{ }^{57}$ Moreover, telephone contact may allow participants to feel relaxed during the interview sessions. Some participants may regard a face-to-face interview as a burden, owing to the need to invite the investigator as a guest in their home.$^{58}$ Also, it has been found that participants are more likely to report sensitive behaviours when the interviewer is not physically present. ${ }^{47}$ Telephone interviewing is a flexible method for the patient, the caregiver and the interviewer. It is a convenient way of arranging individual appointments: the interview can be divided into two parts if the participants need a break, and a telephone interview can be scheduled outside working hours. Finally, the interviewer avoids long transportation time and costs. Although telephone interviews have been noted to be impersonal and not 'the gold standard' for collecting data related to sensitive topics, ${ }^{59}$ the method has many advantages, without existing proof of inferiority. ${ }^{58}$ The participants (patient and the caregiver) are encouraged to carry out the interviews alone so that the conversation is not influenced by the presence of others. However, it will be accepted if the patient needs help/assistance from their caregiver during the interview.

\section{Quantitative data}

Information about the socioeconomic conditions, disease and treatment variables will be obtained by the investigator at baseline. The disease, treatment and clinical variables will be collected throughout the duration of the study, including KPS, which will be obtained by a medical chart review at each test point. Patients will receive three questionnaires by mail at the time of each interview with written instructions to fill out and return in the enclosed stamped addressed envelope. The validated patient-reported questionnaires are (1) Functional Assessment of Cancer Therapy-Brain (FACT-Br), which is an instrument measuring the general QOL and specific symptoms or problems associated with brain cancer. It contains five subscales: physical well-being, social/ family well-being, emotional well-being, functional wellbeing and disease-specific concerns. ${ }^{60}(2)$ The Hospital Anxiety and Depression Scale (HADS) is a short instrument that measures the levels of anxiety and depression, using items that avoid reliance on aspects such as fatigue and insomnia that are common somatic symptoms of illness. It contains two subscales, one measuring anxiety and the other measuring depression. ${ }^{61}(3)$ Physical activity levels will be measured using a single item adapted from Saltin, ${ }^{62}$ where patients rate their physical activity level using four response options "Almost completely inactive': reading, watching TV etc; 'Some physical activity during less than three hours per week': riding a bicycle or walking; 'Regular physical activity for at least $3 \mathrm{~h} /$ week'; and 'Hard physical exercise for more than $4 \mathrm{~h} /$ week'. Additionally, at baseline, patients will also be asked to report retrospectively what their level of activity 
Table 1 Interview topics for patients

Research questions
What happened and how did the
patient experience the time between
the first symptoms and the operation?
Does the patient experience any
symptoms/side effects, and what are
the consequences and how are they
managed?

How are everyday life, work, network and support experienced?

Are there any changes in lifestyle?

How does the patient experience the communication and information with the health professionals?

\section{Does the patient experience}

challenges during everyday activities? What are the needs for rehabilitation? Is the patient motivated to meet her/his needs? Any preferences?

What are the preferences of the patient in relation to support and rehabilitation?

Does the patient seek help from alternative therapy as a supplement to standard treatment?

Are there financial worries and how important are they?

How does the patient prioritise her/his needs?

Is the patient offered help and by whom?

\section{Examples of interview questions}

How did you find out that you were sick? Describe what happened and how did you experience the episode? What has happened since the last interview? How have you been since the last interview?

Do you have any complaints/symptoms/side effects? Does it have any effect on the way that you manage your life? Is there anything that has helped you to overcome the symptoms/side effects? Have you noticed difficulties in remembering, reading or in attention? Have you noticed whether you have changed your way of behaving? How do you cope/manage with this? How is your mood?

Can you describe a typical day? Has it changed from earlier? What do you usually do in your spare time? Do you/can you enjoy yourself now? How would you describe your network? Do you have children? How are they affected? What attitudes/reactions have you experienced to your disease? How is your working situation? Have you changed anything in the way you live your life (eg, diet, exercise, everyday activities, social contacts)

What have the health professionals told you about your disease?

What do you think about that information? Do you lack any information? Have you looked for information-of what kind and where? How do you think about the way the health professionals address you?

Is there anything you find difficult to do?

What would be the best thing that anyone could do for you these days? Do you need any advice on how to handle your situation/condition/symptoms or everyday activities? Are you getting the support you need from the family/hospital/ general practitioner?

Can you imagine being physically active now, and how do you prefer to be active? How many hours a week are you physically active now?

Can you imagine accepting psychological support to handle your situation? Can you imagine getting help to exercise your memory and attention?

What do you do to help yourself? Would you like to participate in physical exercise, cognitive training and/or psychosocial support? (think about preferences, type, location, frequency, duration, supervised or not supervised)

Have you considered any alternative treatment to relieve your situation/condition? Please tell me about your considerations

\section{Are you worried about your financial situation?}

What needs are the most important for you to meet? Have you done anything to solve an unmet need? Do you have suggestions to how we could improve the situation/condition for future patients? Is there any advice you would give other patients being diagnosed with this type of brain tumour? Is there anything else you would like to tell me/or ask me about? was at 3 months prior to diagnosis. The total completion time for filling out all questionnaires is approximately $7-15 \mathrm{~min}$.

\section{Ethical issues}

This study poses no serious ethical problems. The study is registered at The Research Ethics Committees at the Regional capital (33460). According to the Research Ethics Committee, approval is not needed for phase I as it is the non-intervention part of the study; however, ethical approval will be sought for phase II. It has been registered by the Danish Data Protection Agency (2007-58-0015) and will be carried out in accordance with the Declaration of Helsinki. ${ }^{63}$ Written consent will be obtained from each participant, including permission to record the telephone interviews and to use anonymised quotes. Participants will not be identifiable and the authors will disseminate the findings in peerreviewed publications and at relevant conferences.

\section{Data collection and study evaluation}

Participants will be interviewed individually five times over a 12-month period starting at baseline (test 1: time of diagnosis). Test 2 takes place 5-6 weeks postoperatively, which is approximately 2 weeks after initiation of the oncological treatment. Test 3 is at 28 weeks (after first treatment response scan), test 4 is at 40 weeks (after second treatment response scan) and the final test (test 
Table 2 Interview topics for caregivers

Research questions
How did you experience the period
between the first symptoms and the
operation?

Does the caregiver experience that the patient has any symptoms/side effects, what are the consequences and how are they managed?

How is everyday life, network and support?

What norms and attitudes do the caregivers experience?

Are there any changes in behaviour?

How does the caregiver experience the communication and information with the health professionals?

Does the caregiver experience any challenges in the physical environment?

What are the needs of rehabilitation? Is the caregiver motivated to meet her/ his own needs?

Any preferences?

What are the preferences of the caregivers in relation to support and rehabilitation?

Does the caregiver seek help from alternative methods as a supplement to standard treatment?

Are there financial worries and how important are they?

How does the caregiver prioritise her/ his own needs?

\section{Examples of interview questions}

How did you find out that $X$ was sick?

Describe what you observed, what happened and how you did experience that period? How was it to be a caregiver while $X$ was admitted to hospital? What has happened since the last interview?

How would you describe what $X$ experiences these days? Do you observe any symptoms/side effects? Does it have any effect on the way you live your life? Is there anything that has helped you to manage X's symptoms/side effects?

Have you observed any difficulties in remembering, reading or attending for $X$ ? Has he/she changed personality/way of behaving? How does that affect you?

Have you ever thought that brain cancer differs from other types of cancer? Please tell me about this

Can you describe a typical day? Has that changed from earlier? What do you usually do during your spare time? Do you enjoy yourself now? Are there family/ friends that have special meaning to you after $X$ got sick? How? Do you have children? How are they affected? How would you describe your network? What attitudes/reactions to the disease, do you meet?

How is your working situation right now? Have you changed anything in the way you live your life (eg, diet, every day activities)?

What have the health professionals told you about X's disease?

Did you receive the right amount of information? How did you find the written information? Do you lack any information? Have you looked for information, of what kind and where? How do you find the way the health professionals address you? Do you experience practical problems in your life after $X$ became diseased? Have you taken on another role since $X$ became sick? How does it affect you?

What would be the best thing someone can do for you right now? Do you need any advice on how to handle your situation? Are you getting the support you need from family/hospital/general practitioner?

Is this a stressful period for you? Do you experience any symptoms, physical problems?

What do you do to help yourself (physical, social and psychological activities)? Would you like to participate in a support group/information group? Think about preferences, type, location, frequency, duration, supervised or not supervised support

Have you considered any alternative treatment to relieve your or X's situation/ condition?

Are you worried about your financial situation now that $\mathrm{X}$ is sick?

What needs are most important for you to meet? Have you done anything to solve an unmet need? Do you have suggestions on how we could improve the situation/ condition in the future for caregivers and for patients with a brain tumour? Is there any advice you would give to other caregivers? Is there anything else you would like to tell me/or ask me about?
$5)$ is at 52 weeks. It is the aim that both tests 3 and 4 should take place approximately 1 week after the results of the response scan are known by the patient and the caregiver. The five test points (figure 1) are selected in order to study the variations in needs along the treatment trajectory. ${ }^{64}$ The patients will be asked to complete questionnaires at all five test points. The patient and the caregiver will be interviewed separately within the same week in order to seek perspective on their life situation within the same time frame. A final interview will be conducted with the caregiver in case the patient dies.
This interview will be scheduled approximately 2 months postbereavement.

\section{ANALYSIS}

The semistructured interviews will be recorded and transcribed in full length by either the interviewer or a research assistant. Interview data will be transferred to the NVivo V.9 software program. ${ }^{65}$ This program allows transparency in the process from raw data (interview transcripts) to comprehensive understanding, which 
increases the credibility and reliability of the findings. ${ }^{66}$ Two researchers will conduct the analysis in order to strengthen the validity of the study. ${ }^{59}$ Qualitative data from the interviews at each test time point will be analysed independently at each time point and then analysed across all interview tests time points within the group of patients and within the group of caregivers. The findings from the patient's perspective will be viewed in parallel to the perspectives of the caregivers.

A thematic analysis will be conducted for the analysis of the semistructured interviews. Thematic analysis is a basic method for qualitative analysis as it identifies, analyses and report themes and patterns within the data. ${ }^{67}$ The following section describes the procedure that will be applied in this study. The findings will be presented in themes and subthemes. A theme is an expression or context being defined as important by the researchers. In this study, a theme is not necessarily dependent on quantifiable measures, but rather captures an important meaning in relation to the research questions and aims. ${ }^{67}$

Data from the interviews are descriptive in nature and reflect the subjective experience of the life situation of patients with HGG and their caregivers. The investigator will conduct a pilot test of the first two interviews. The steps for thematic analysis are inspired by Braun and Clarke $^{67}$ and are as follows: Step 1: data are transcribed immediately after an interview by the investigator or a research assistant. Transcriptions are read several times until the investigators are familiar with the depth and extent of the content. This process guides the investigators to the next level of analysis. Step 2: the text is read and divided into units of meanings, which are identified by the investigators. Some meaning units are sentences, others a whole paragraph. In this process, data are coded in order to organise them into meaningful groups. This process assures that the whole dataset is systematically worked through by the investigators. Step 3: the codes are then analysed in order to consider if different codes can be combined into mutual themes. This three-step process will result in a collection of themes and subthemes.

Socioeconomic information and data from questionnaires will be entered into a database and subscale scores computed according to guidelines in questionnaire manuals. Procedures for the data entry and audit program are established and written to ensure correct data keying. Quantitative data from questionnaires and KPS constitute using repeated measurements, and linear mixed models to analyse them. KPS, each of the subscales of FACT-Br, the two subscales of HADS and the responses to the ordinal item about leisure time physical activity will be analysed separately. The mean values and corresponding $95 \%$ confidence limits will be computed and plotted along with the individual trajectories. No significance testing will be performed; rather, the development in each of the subscales of the questionnaires will be compared with the development in KPS and the results from the qualitative analysis.

\section{SUMMARY}

The aim of this paper was to describe the design and methods of an upcoming qualitative and quantitative longitudinal research study. Interviews with 30 patients and 30 caregivers will provide information regarding their experiences during the first year after diagnosis of an HGG. Quantitative measurements of QOL, emotional well-being and physical activity provide additional information. The data will create the basis for a rehabilitation programme regarding the content and timing of such supportive, rehabilitative and palliative interventions.

The overall aim was to develop a tailored and multidimensional rehabilitation programme for patients with HGG and their caregivers. The key to good clinical care is to understand what is important to the patients and their caregivers during various phases of the cancer trajectory. Interventions that target support, information, communication and improve physical and cognitive function in daily life can be important to facilitate a reduced feeling of distress and to increase QOL. This study seeks to support future evidence for guidelines regarding the supportive, rehabilitative and palliative interventions for patients with HGG and their caregivers.

\section{Author affiliations}

${ }^{1}$ Department of Neurosurgery, The University Hospital of Copenhagen, Rigshospitalet, Copenhagen, Denmark

${ }^{2}$ The University Hospitals Center for Health Science Research (UCSF), Center for Integrated Rehabilitation for patients with Cancer (CIRE), Copenhagen,

Denmark

${ }^{3}$ The University Hospital of Copenhagen, Rigshospitalet, Neuroscience Center, Copenhagen, Denmark

${ }^{4}$ Department of Public Health, Section of Biostatistics, University of Copenhagen, Copenhagen, Denmark

${ }^{5}$ Department of Clinical Medicine, Section of Neurology, Psychiatry and Sensory Sciences, The University of Copenhagen, Copenhagen, Denmark

Contributors KP, MJA, JKJ, KBC and MJU were involved in the contribution and design of the study, KP participated in the drafting of the article and revising for important intellectual content. All authors have read and approved the final manuscript.

Funding The study is supported by grants from The Center for Integrated Rehabilitation of Cancer patients (CIRE), a centre established and supported by The Danish Cancer Society and The Novo Nordisk Foundation. Furthermore, it is supported by The Neuro Center at Copenhagen University Hospital; Rigshospitalet in Denmark, The Novo Nordisk Foundation, The Capital Regional Research Foundation, Torben and Alice Frimodts Foundation, Vera and Flemming Westerbergs Foundation and Hetland Olsen's Foundation.

Competing interests None.

Ethics approval Study was registered in 2012 at The Research Ethics Committees at the Regional capital under the number 33460 . However, the Research Ethics Committee responded that approval is not needed before the intervention study will be developed. At that time the researcher will seek ethical approval. Study has been approved by the Danish Data Protection Agency (2007-58-0015). The study will be carried out in accordance with the Declaration of Helsinki (WHO, 2000).

Provenance and peer review Not commissioned; externally peer reviewed.

\section{REFERENCES}

1. Stupp R, Gander M, Leyvraz S, et al. Current and future developments in the use of temozolomide for the treatment of brain tumours. Lancet Oncol 2001;2:552-60. 
2. Starkweather AR, Sherwood P, Lyon DE, et al. A biobehavioral perspective on depressive symptoms in patients with cerebral astrocytoma. J Neurosci Nurs 2011;43:17-28.

3. Wen PY, Kesari S. Malignant gliomas in adults. N Engl $J$ Med 2008;359:492-507.

4. Stupp R, Hegi ME, Mason WP, et al. Effects of radiotherapy with concomitant and adjuvant temozolomide versus radiotherapy alone on survival in glioblastoma in a randomised phase III study: 5-year analysis of the EORTC-NCIC trial. Lancet Oncol 2009; 10:459-66.

5. Hart MG, Grant R, Garside R, et al. Chemotherapy wafers for high grade glioma. Cochrane Database Syst Rev 2011;(3):CD007294.

6. Lucas MR. Psychosocial implications for the patient with a high-grade glioma. J Neurosci Nurs 2010;42:104-8.

7. Klein M, Taphoorn MJ, Heimans JJ, et al. Neurobehavioral status and health-related quality of life in newly diagnosed high-grade glioma patients. J Clin Oncol 2001;19:4037-47.

8. Taphoorn MJ, Klein M. Cognitive deficits in adult patients with brain tumours. Lancet Neurol 2004;3:159-68.

9. Douw L, Klein M, Fagel SS, et al. Cognitive and radiological effects of radiotherapy in patients with low-grade glioma: long-term follow-up. Lancet Neurol 2009;8:810-18.

10. Byrne TN. Cognitive sequelae of brain tumor treatment. Curr Opin Neurol 2005;18:662-6.

11. Behin A, Delattre JY. Complications of radiation therapy on the brain and spinal cord. Semin Neurol 2004;24:405-17.

12. Armstrong $\mathrm{CL}$, Stern $\mathrm{CH}$, Corn BW. Memory performance used to detect radiation effects on cognitive functioning. Appl Neuropsychol 2001;8:129-39.

13. Kosteljanetz M. Brain tumors. Mainly glial tumors in adults. Ugeskr Laeger 2002;164:3017-20.

14. Armstrong TS, Cohen MZ, Eriksen LR, et al. Symptom clusters in oncology patients and implications for symptom research in people with primary brain tumors. J Nurs Scholarsh 2004;36:197-206.

15. Ownsworth T, Chambers S, Hawkes A, et al. Making sense of brain tumour: a qualitative investigation of personal and social processes of adjustment. Neuropsychol Rehabil 2011;21:117-37.

16. Molassiotis A, Wilson B, Brunton L, et al. Symptom experience in patients with primary brain tumours: a longitudinal exploratory study. Eur J Oncol Nurs 2010;14:410-16.

17. Kim BR, Chun MH, Han EY, et al. Fatigue assessment and rehabilitation outcomes in patients with brain tumors. Support Care Cancer 2011;20:805-12.

18. Klein M, Engelberts $\mathrm{NH}$, Van der Ploeg HM, et al. Epilepsy in low-grade gliomas: the impact on cognitive function and quality of life. Ann Neurol 2003:54:514-20.

19. Struik K, Klein M, Heimans JJ, et al. Fatigue in low-grade glioma. $J$ Neurooncol 2009;92:73-8.

20. Lovely MP. Symptom management of brain tumor patients. Semin Oncol Nurs 2004;20:273-83.

21. Taphoorn MJ, Stupp R, Coens C, et al. Health-related quality of life in patients with glioblastoma: a randomised controlled trial. Lancet Oncol 2005;6:937-44.

22. Taphoorn MJ, Van den Bent MJ, Mauer ME, et al. Health-related quality of life in patients treated for anaplastic oligodendroglioma with adjuvant chemotherapy: results of a European Organisation for Research and Treatment of Cancer randomized clinical trial. J Clin Oncol 2007:25:5723-30.

23. Mauer M, Stupp R, Taphoorn MJ, et al. The prognostic value of health-related quality-of-life data in predicting survival in glioblastoma cancer patients: results from an international randomised phase III EORTC Brain Tumour and Radiation Oncology Groups, and NCIC Clinical Trials Group study. Br J Cancer 2007;97:302-7.

24. Giovagnoli AR, Silvani A, Colombo E, et al. Facets and determinants of quality of life in patients with recurrent high grade glioma. $J$ Neurol Neurosurg Psychiatry 2005;76:562-8.

25. Jones LW, Friedman AH, West MJ, et al. Quantitative assessment of cardiorespiratory fitness, skeletal muscle function, and body composition in adults with primary malignant glioma. Cancer 2010;116:695-704.

26. Salo J, Niemela A, Joukamaa M, et al. Effect of brain tumour laterality on patients' perceived quality of life. J Neurol Neurosurg Psychiatry 2002;72:373-7.

27. Ownsworth T, Hawkes A, Steginga S, et al. A biopsychosocial perspective on adjustment and quality of life following brain tumor: a systematic evaluation of the literature. Disabil Rehabil 2009;31:1038-55.

28. Brown PD, Ballman KV, Rummans TA, et al. Prospective study of quality of life in adults with newly diagnosed high-grade gliomas. $J$ Neurooncol 2006;76:283-91.
29. Giovagnoli AR. Quality of life in patients with stable disease after surgery, radiotherapy, and chemotherapy for malignant brain tumour. J Neurol Neurosurg Psychiatry 1999;67:358-63.

30. Recht L, Glantz M, Chamberlain M, et al. Quantitative measurement of quality outcome in malignant glioma patients using an independent living score (ILS). Assessment of a retrospective cohort. J Neurooncol 2003;61:127-36.

31. Ruden E, Reardon DA, Coan AD, et al. Exercise behavior, functional capacity, and survival in adults with malignant recurrent glioma. $J$ Clin Oncol 2011;29:2918-23.

32. Deutsch MB, Panageas KS, Lassman AB, et al. Steroid management in newly diagnosed glioblastoma. $J$ Neurooncol 2013;113:111-16

33. Hempen C, Weiss E, Hess CF. Dexamethasone treatment in patients with brain metastases and primary brain tumors: do the benefits outweigh the side-effects? Support Care Cancer 2002;10:322-8.

34. Nahaczewski AE, Fowler SB, Hariharan S. Dexamethasone therapy in patients with brain tumors-a focus on tapering. $J$ Neurosci Nurs 2004:36:340-3.

35. Horber FF, Hoopeler H, Scheidegger JR, et al. Impact of physical training on the ultrastructure of midthigh muscle in normal subjects and in patients treated with glucocorticoids. $J$ Clin Invest 1987;79:1181-90.

36. Madsen K, Poulsen HS. Needs for everyday life support for brain tumour patients' relatives: systematic literature review. Eur $J$ Cancer Care (Engl) 2011;20:33-43.

37. Spetz A, Henriksson R, Salander P. A specialist nurse as a resource for family members to patients with brain tumors: an action research study. Cancer Nurs 2008;31:E18-26.

38. Wideheim AK, Edvardsson T, Pahlson A, et al. A family's perspective on living with a highly malignant brain tumor. Cancer Nurs 2002;25:236-44

39. Adelbratt $S$, Strang $P$. Death anxiety in brain tumour patients and their spouses. Palliat Med 2000;14:499-507.

40. Strang S, Strang P. Spiritual thoughts, coping and 'sense of coherence' in brain tumour patients and their spouses. Palliat Med 2001;15:127-34.

41. Strang S, Strang P, Ternestedt BM. Existential support in brain tumour patients and their spouses. Support Care Cancer 2001;9:625-33.

42. Sherwood PR, Donovan HS, Given CW, et al. Predictors of employment and lost hours from work in cancer caregivers. Psychooncology 2008;17:598-605.

43. Keir ST, Guill AB, Carter KE, et al. Stress and intervention preferences of patients with brain tumors. Support Care Cancer 2006;14:1213-19.

44. Sherwood PR, Given BA, Given CW, et al. Predictors of distress in caregivers of persons with a primary malignant brain tumor. Res Nurs Health 2006;29:105-20.

45. Hricik A, Donovan $\mathrm{H}$, Bradley SE, et al. Changes in caregiver perceptions over time in response to providing care for a loved one with a primary malignant brain tumor. Oncol Nurs Forum 2011;38:149-55.

46. McConigley R, Halkett G, Lobb E, et al. Caring for someone with high-grade glioma: a time of rapid change for caregivers. Palliat Med 2010;24:473-9.

47. Moore G, Collins A, Brand C, et al. Palliative and supportive care needs of patients with high-grade glioma and their carers: a systematic review of qualitative literature. Patient Educ Couns 2013:91:141-53.

48. Creswell J, Clark V. ed. Designing and conducting mixed methods research. 2nd edn. SAGE, 2011.

49. Murray SA, Kendall M, Carduff $E$, et al. Use of serial qualitative interviews to understand patients' evolving experiences and needs. BMJ 2009;339:b3702

50. Lobb EA, Halkett GK, Nowak AK. Patient and caregiver perceptions of communication of prognosis in high grade glioma. $J$ Neurooncol 2011:104:315-22.

51. Walker M, Brown J, Brown $\mathrm{K}$, et al. Practical problems with the collection and interpretation of serial quality of life assessments in patients with malignant glioma. J Neurooncol 2003;63:179-86.

52. Polit D, Beck C. ed. Nursing research. Generating and assessing evidence for nursing practice. 9th edn. Wolters Kluwer: Lippincottt Williams \& Wilkins, 2012.

53. Kvale S. InterView. Copenhagen: Hans Reitzels Forlag, 1997.

54. Galesic M, Tourangeau R, Couper MP. Complementing random-digit-dial telephone surveys with other approaches to collecting sensitive data. Am J Prev Med 2006;31:437-43.

55. Trier-Bieniek A. Framing the telephone interview as a participant-centered tool for qualitative research: a methodological discussion. Qual Res 2012;12:630- 44. 
56. Stephens S. Collecting data from elites and ultra elites: telephone and face-to-face interviews with macroeconomists. Qual Res 2007;7:203-16.

57. Sturges J, Hanrahan K. Comparing telephone and face-to-face qualitative interviewing: a research note. Qual Res 2004;4:107-18.

58. Novick $\mathrm{G}$. Is there a bias against telephone interviews in qualitative research? Res Nurs Health 2008(31):391-8.

59. Polit D, Beck C,. eds. Nursing research: principles and methods. 7th edn. Lippincott Williams \& Wilkins, 2004.

60. Weitzner MA, Meyers CA, Gelke CK, et al. The Functional Assessment of Cancer Therapy (FACT) scale. Development of a brain subscale and revalidation of the general version (FACT-G) in patients with primary brain tumors. Cancer 1995;75:1151-61.

61. Snaith RP. The hospital anxiety and depression scale. Health Qual Life Outcomes 2003;1:29.
62. Saltin B, Grimby G. Physiological analysis of middle-aged and old former athletes. Comparison with still active athletes of the same ages. Circulation 1968;38:1104-15.

63. WHO. Helsinki Declaration. 2000.

64. Halkett GK, Lobb EA, Oldham L, et al. The information and support needs of patients diagnosed with High Grade Glioma. Patient Educ Couns 2010;79:112-19.

65. Skorkjær Binderkrantz A, Bøgh Andersen L. Guide til NVivo9/Guide to NVivo9. 1st edn. Hans Reitzels, 2011.

66. Dreyer PS, Steffensen BF, Pedersen BD. Life with home mechanical ventilation for young men with Duchenne muscular dystrophy. $J \mathrm{Adv}$ Nurs 2010;66:753-62.

67. Braun V, Clarke V. Using thematic analysis in psychology Qual Res Psychol 2006;3:77-101. 